\title{
An atomic spectrum recorded with a single-molecule light source
}

\author{
Wilhelm Kiefer $^{1} \cdot$ Mohammad Rezai $^{1} \cdot$ Jörg Wrachtrup $^{1,2} \cdot$ Ilja Gerhardt $^{1,2}$
}

Received: 31 July 2015 / Accepted: 7 October 2015 / Published online: 19 February 2016

(c) The Author(s) 2016. This article is published with open access at Springerlink.com

\begin{abstract}
A single molecule under cryogenic conditions allows one to realize an extremely bright and simultaneously narrow-band single-photon source. We present a review on the different excitation schemes of a single molecule and present the corresponding single-photon nature of the emitted light. Single-molecule spectroscopy has been recently interlinked with atomic spectroscopy. This optical interconnect among the different quantum systems might be enhanced by a so-called Faraday anomalous dispersion optical filter-an ideal tool for many experiments in quantum optics. We introduce our theoretical and experimental approach on these filters which are based on hot atomic sodium vapor. The electrical tunability together with the brightness of a single molecule allows us to record a full atomic spectrum of this filter with the single photons originating from a single-molecular emitter.
\end{abstract}

\section{Introduction}

The efficient generation of single photons is a central point of research in the past two decades. Their applications span answering fundamental physics questions, quantum

This paper is part of the topical collection "Quantum Repeaters: From Components to Strategies" guest edited by Manfred Bayer, Christoph Becher and Peter van Loock.

Ilja Gerhardt

i.gerhardt@fkf.mpg.de

1 3. Physikalisches Institut, Universität Stuttgart and Stuttgart Research Center of Photonic Engineering (SCoPE) and IQST, Pfaffenwaldring 57, Stuttgart 70569, Germany

2 Max Planck Institute for Solid State Research, Heisenbergstraße 1, 70569 Stuttgart, Germany communication, quantum information, and the generation of sub-shot noise light sources. Numerous experimental implementations have been realized to generate single photons. One of them is the use of a single emitter, which, once excited, can only emit a single photon per unit time. Such sources principally allow for a deterministic single-photon generation. This implies that the emitter is excited, e.g., with an optical $\pi$-pulse and then emits subsequently a single photon on demand [1].

Historically, the first anti-bunched single-photon source was a diluted atomic beam [2]. Thereafter, the technique of trapping single ions has been established [3], allowing for numerous experiments with single photons and addressing fundamental questions of light-matter interaction $[4,5]$. In a solid-state environment, the excitation and the collection efficiency to and from a single emitter can be significantly higher $[6,7]$. This is partially accounted simply to a reduced distance of the emitter to the first lens. In a vacuum chamber and with an assumed point-like emission pattern of an atom under study, the collection efficiency to the first lens is solely given by the solid angle of the detection optics. This has been optimized in the past [8,9], but already some millimeter distance reduce this to a small fraction of the entire emission. Therefore, a solid-state source can be more efficient when the first lens is much closer to the emitter than in the above-named case. Furthermore, the emission pattern is altered when the emitter experiences a certain change in refractive indices in its local proximity [10]. Already a simple, three-layered system allows for the efficient collection of light from a solid-state system and might reach up to unity efficiency [11]. Another option is the tailored emission into a defined mode by specialized structures, such as nano-pillars $[12,13]$. Although the research on single emitters in a solidstate environment addresses many more questions on highresolution microscopy [14] or nanoscopic sensing [15], this paper focuses on single emitters as single-photon sources. 
Despite the limited emission rate of a single atom in a vacuum environment, numerous important experiments have been performed when such photons are interlinked with further quantum nodes such as other atoms [16, 17]. There, the emission is enhanced with a Fabry-Pérot cavity. Only recently, it became possible to interlink photons from a single solid-state emitter with an atomic system [18-20]. These experiments have the potential to be utilized in combined experiments with single photons and atoms, but the long-term stability and the versatility of the sources has not been directly proven so far.

In this paper, we review the spectral properties of single molecules and how these can be used as single-photon emitters for an optical interface to atomic systems. The different excitation options of these molecules are discussed, and measurements on the way to an efficient atom-resonant single-photon source are presented. A single molecule, which does not show any blinking and bleaching under cryogenic conditions, shows such a versatile nature, such that it is possible to record an atomic spectrum with the introduced single-photon source.

\section{Single-molecule studies under cryogenic conditions}

Certain organic dye molecules can be treated as optically accessible two-level systems at liquid helium temperatures. The low temperature suppresses a coupling to the external phonon bath, such that the electronic transition on the zerophonon line (ZPL) can be lifetime-limited. The relation between the longitudinal $\left(T_{1}\right)$ and transversal $\left(T_{2}\right)$ relaxation time, as $1 / T_{2}=1 /\left(2 \cdot T_{1}\right)$ holds.
The selection of molecules is limited usually to rigid molecules, which exhibit a Franck-Condon factor close to unity. This implies no severe change in the nuclear core coordinates in the excited state and a small Stokes shift of the absorption versus the emission spectrum. The poly-aromatic hydrocarbons (PAHs) are usually good candidates, and early experiments on single molecules were performed on pentacene (CAS: 135-48-8) [21, 22]. Due to the low quantum yield of pentacene, several other molecules were explored thereafter. The candidates terrylene (CAS: 18872-7) [23] and its derivates [24, 25], perylene (CAS: 19855-0) [26, 27], and 2.3,8.9-dibenzanthanthrene (CAS: 18842-1, see Fig. 1b) [28, 29] have to be named in this context. This list is not complete, and more molecules can be used at cryogenic conditions [30, 31].

In many experiments, the molecules under study are frozen into an organic matrix, such as the alkanes. The system forms a so-called Shpol'skiı̌-matrix. Other options are possible, such as single molecules in rare gas hosts [32] or in crystalline structures $[23,33]$. The exact spectral properties such as linewidth and transition wavelength are strongly dependent on the host matrix. The matrix also influences the spectral stability of the system under study. Spectral diffusion or spectral jumps might be disadvantageous for single-photon generation. This is luckily suppressed in the presented molecule/host matrix system.

The data for this study are the spectral study on one single dibenzanthanthrene (DBATT) molecule embedded in $n$-tetradecane. The study was performed in a helium bath cryostat, while the sample was immersed in super-fluid helium. The base temperature reached down to $1.4 \mathrm{~K}$. All presented molecular spectra are recorded on one individual molecule under different continuous-wave (cw) excitation
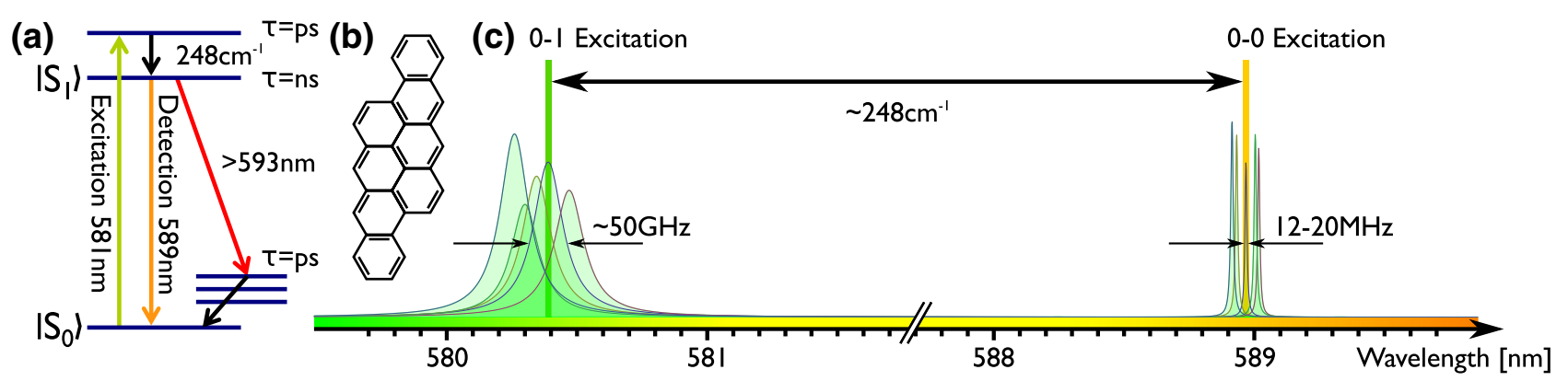

Fig. 1 a Simplified Jabłoński diagram of a single molecule under cryogenic conditions. The electronic states are singlet states and are labeled as $\left|S_{n}\right\rangle$. b Single-molecule structure of 2.3,8.9-dibenzanthanthrene, DBATT, chemical formula: $\mathrm{C}_{30} \mathrm{H}_{16}$. c Schematic spectral representation of the level diagram. Thick vertical lines represent the possible spectral positions for laser excitation. The higher vibrational level of the electronic excited state has an energy difference of $248 \mathrm{~cm}^{-1}$ above the electronic excited and its vibrational ground state. The longitudinal lifetime of these vibrational levels is in the picosecond range, such that the linewidth is several GHz.

This implies that a spectral selection of single molecules in the zerophonon-line excitation is very easy, while the excitation into higher vibrational states might lead to an increased background due to the excitation of other molecules. Height of the levels is not to scale. After a decay into the electronic excited state, the system relaxed by emitting single photons. The emitted spectrum of a decay into the ground state depends on the branching ratio into the ground state and into the vibrational levels, which is approx. 50:50 for dibenzanthanthrene 
schemes. Due to the fact that the intersystem crossing rate is small, we do not observe any (triplet-)blinking throughout our studies.

Figure 1a shows a reduced Jabłoński diagram, in which the relevant levels are shown. The electronic levels of the molecule are labeled as $\left|S_{0}\right\rangle$ and $\left|S_{1}\right\rangle$. As usual for organic molecules, the states are singlet states and exhibit no further state splitting, with, for example, optical addressable spins. In the diagram, we omit the triplet levels, the $\left|T_{0}\right\rangle$ state, since they are not relevant for the extraction of photons in a single molecule. The direct transition between the two singlet levels is called the zero-phonon transition, since no phonons are excited in the system. To the first approximation, this first electronic transition (from $\left|S_{0}\right\rangle$ to $\left|S_{1}\right\rangle$ ) represents a transition from the highest occupied molecular orbital to the lowest unoccupied molecular orbital, a so-called HOMO-LUMO transition. Each of the electronic levels has higher vibrational states. Therefore, an excited molecule has not only the possibility to decay directly into the electronic ground state, but also the ability to emit a red-shifted photon and decay into a vibrational state of the electronic ground state. These levels are short-lived (lifetime approx. ps), and the system decays with no further photonic emission to the vibrational and electronic ground state. Due to the similarity of the electronic levels $\left|S_{0}\right\rangle$ and $\left|S_{1}\right\rangle$, also the vibrational levels are comparable. This implies that the system can also be excited into a higher vibrational level of the electronic excited state. This level displays a comparable energy difference to the zero-phonon state in both cases. The exact excitation schemes are explained hereafter.

Figure 1c shows the corresponding transitions of a single molecule on a wavelength scale. The common way to perform a study on single molecules under cryogenic conditions is an excitation directly between the electronic states. This zero-phonon line is extremely narrow band and reaches with the presented molecule (dibenzanthanthrene) down to a linewidth of $12.5 \mathrm{MHz}$. For this so-called 0-0 excitation scheme, the concentration of molecules in the sample under research can be very high, and it might be that several molecules are located inside the excitation laser focus. Individual molecules can then be simply spectralselected, since different molecules experience a different nano-environment and therefore a different electronic transition energy. This is despite the fact that the molecules are chemically identical. This spectral distribution corresponds to the inhomogeneous broadening of many molecules inside a solid-state sample.

Another way to excite a single molecule is the excitation into a higher vibrational level of the electronic excited state. The molecule will then decay without the emission of a photon into the electronic excited, but vibrational ground state (Kasha's rule) and decay with the emission of a photon only then. This scheme is indicated by the arrows in Fig. 1a. Since the lifetime of the vibrational state is in the picosecond range, the systems spectral linewidth depends on the electronic lifetime of the $\left|S_{1}\right\rangle$-state. The branching ratio between a direct decay into the $\left|S_{0}\right\rangle$-state and a phonon-mediated (Stokes-shifted) emission is for the introduced DBATT molecule about $50 \%$.

Figure 2 shows experimental fluorescence excitation spectra in the two different excitation schemes. The cw laser is spectral-tuned across the transition of a single molecule. Only when the molecule is in resonance, the cycle of excitation by the laser and subsequent single-photon emission by the molecule goes on. Both spectra represent raw data and are simply fitted with a Lorentzian function. Figure 2a shows the resonant 0-0 excitation. All red-shifted fluorescence is detected behind a long-pass filter (Semrock 594LP). The determined linewidth represents the narrowest
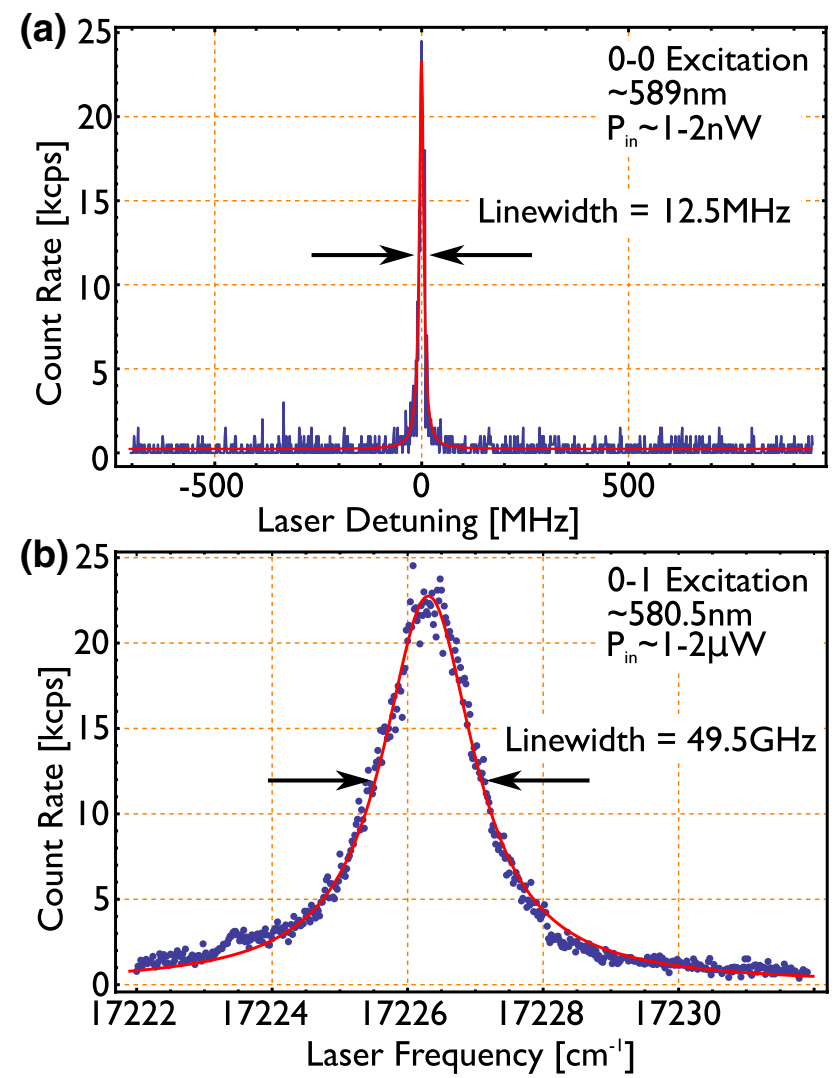

Fig. 2 a Fluorescence excitation spectrum of a single molecule into the zero-phonon line of the electronic excited state. Detection of redshifted photons, which are emitted when the molecule decays into the vibrational levels of the ground state. This is the so-called 0-0 excitation. b Fluorescence excitation spectrum of a single molecule into a higher vibrational level of the electronic excited state. The linewidth is lifetime-limited. This scheme is called " $0-1$ excitation". Detection of the narrow-band emission around $589 \mathrm{~nm}$. This is the direct decay into the electronic ground state. The spectrum corresponds to the spectral linewidth in a 
measured one our laboratories and amounts to $12.5 \mathrm{MHz}$. This corresponds well to the value, which is given in the literature [28]. The background emission represents the dark count level of the utilized single-photon detector (Excellitas, SPCM-AQR). The excitation power, measured in front of the cryostat, was on the order of $1 \mathrm{nW}$. This spectrum, acquired at the low excitation limit of the system, shows no high-power broadening, which would correspond to the behavior of a two-level system. This broadening can be described by the equation $\Gamma_{\text {eff }}^{2}=\Gamma_{2}^{2}+\Omega_{\text {Rabi }}^{2} \frac{\Gamma_{2}}{\Gamma_{1}}$, with $\Gamma_{1}=1 / T_{1}$ and $\Gamma_{2}=1 / T_{2}$, see, for example, [34]. Commonly, spectral linewidth between 12.5 and $20 \mathrm{MHz}$ is measured for isolated DBATT molecules in $n$-tetradecane. This line can be resonant with the sodium $D_{1}$-line or the sodium $D_{2}$-line.

The 0-1 excitation, shown in Fig. $2 b$, shows a scan of the laser over the transition between the ground state, $\left|S_{0}\right\rangle$, and the first electronic and first vibrational excited state. The wavelength is approx. $580.5 \mathrm{~nm}$. After a few experimental runs, the exact frequency was known and the experiments could directly be started with $0-1$ excitation. In our experimental configuration, only the narrow-band photons from the direct decay of the $\left|S_{1}\right\rangle$-state are detected. These photons show a spectral linewidth corresponding to the measured one for the 0-0 excitation scheme as described above. In this case, the photons are $12.5 \mathrm{MHz}$ wide. Since the vibrational level decays in the picosecond range, the excitation linewidth is increased roughly by three orders of magnitude and amounts to $49.5 \mathrm{GHz}\left(1 \mathrm{~cm}^{-1}=30 \mathrm{GHz}\right)$. The excitation intensity of the molecule is also increased by the same factor.

This experimental set of data shows another crucial problem of the study with both excitation schemes: The spectral selection of a single molecule under resonant 0-0 excitation is very good. A few $\mathrm{MHz}$ detuning allows to reduce the contribution of a single molecule by orders of magnitude. For the 0-1 excitation, a small detuning of the laser does not strongly reduce the emission of the molecule. As a rule of thumb, the concentration of molecules for $0-1$ excitation has to be smaller by a factor of 1000-otherwise several molecules will spectral overlap. This problem is also depicted in Fig. 1c. We fine-tuned the concentration such that we have approx. 1-5 molecules in a $50 \times 50 \mu \mathrm{m}^{2}$ wide scan under 0-0 excitation at one specific laser frequency, which was commonly set to be resonant to atomic sodium.

The proof whether single photons are emitted by a single molecule under study is obtained by recording an autocorrelation function. The presence of single photons leads to a so-called anti-bunched and sub-Poissonian photon statistics. This autocorrelation function is mathematically expressed as:

$g^{(2)}(\tau)=\frac{\langle I(t) I(t+\tau)\rangle}{\langle I(t)\rangle^{2}}$
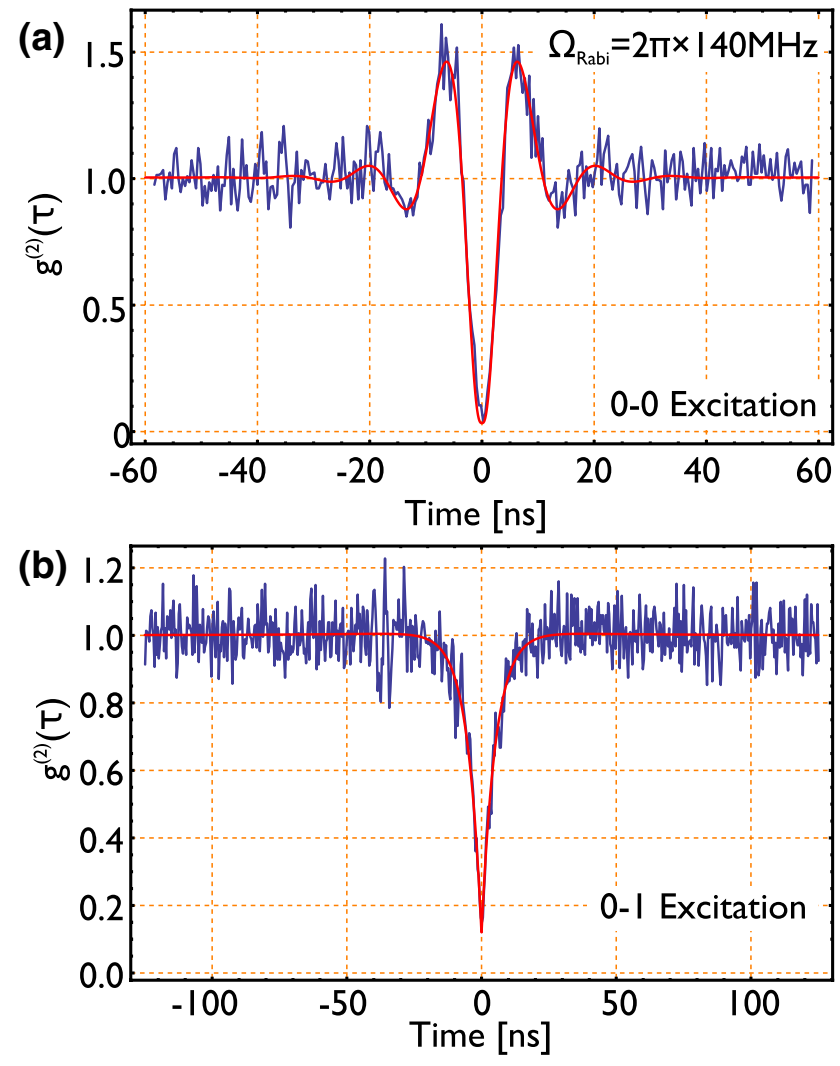

Fig. 3 Photon anti-bunching under different excitation schemes. a Uncorrected recording of the photon statistics when the molecule is excited resonantly into the zero-phonon line. Due to the strong optical interaction of the system to the driving field $(430 \mathrm{nW}$ in front of the cryostat), Rabi oscillations occur. Fit by a simple coherently driven two-level-system. b Uncorrected measurement of the anti-bunching under 0-1 excitation (raw data, $37 \mu \mathrm{W}$ in front of the cryostat). The characteristic lifetime $(\approx 10 \mathrm{~ns})$ is dominated by the longitudinal lifetime of the electronic excited and vibrational ground state. This is an indication for the spectral linewidth of the emitted photons. The fit (red) represents the fit of a two-level system

We like to denote that the function is acquired in a "start-multiple-stop" measurement, i.e., from every photon on a detector in a Hanbury Brown and Twiss configuration, the delay times to all other photons on the other detector up to a certain recording time are determined. This is performed by recording all photon arrival times and processing the interphoton waiting times. This histogram gives to a good approximation (dead time, jitter, etc.), the $g^{(2)}(\tau)$-function as denoted in Eq. 1. Due to the $T_{1}$ time of the system of about $10 \mathrm{~ns}$, such technical issues, e.g., electrical jitter in the detector, are of minor importance, since these issues are much smaller. Subsequently, we are able to plot the raw, uncorrected data in Fig. 3.

Both excitation schemes lead to different $g^{(2)}(\tau)$-functions. In the case of resonant excitation, the system undergoes Rabi oscillations. This is already observed in the first reports on single molecules [35]. Mathematically, the system needs to be treated by a full solution for the two-level 


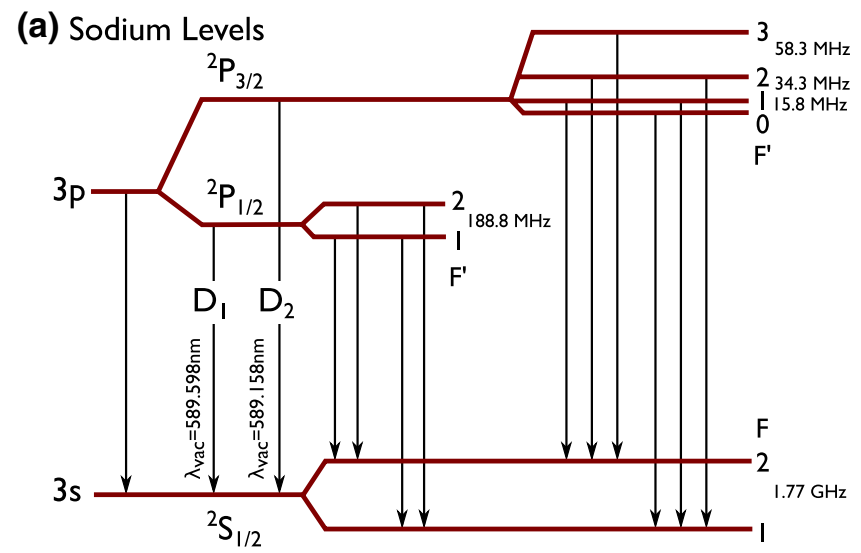

Fig. 4 a Relevant optical sodium transitions. Usually, our experiments are performed on the $D_{2}$-line, due to the higher oscillator strength. b When the system is in a magnetic field, and the net electric susceptibilities are calculated, all levels have to be taken into

system, including the coherences. This is usually performed by assuming a quantum master equation by a Lindblad superoperator. The final equation, including the coherences, reads:

$$
\begin{aligned}
g^{(2)}(\tau)= & 1-\mathrm{e}^{-\frac{1}{2}|\tau|(\Gamma 1+\Gamma 2)} . \\
& \left(\frac{(\Gamma 1+\Gamma 2) \sin \left(|\tau| \Omega_{\mathrm{Rabi}}\right)}{2 \Omega_{\mathrm{Rabi}}}+\cos \left(|\tau| \Omega_{\mathrm{Rabi}}\right)\right) .
\end{aligned}
$$

This equation is then used to fit the experimental data in Fig. 3a.

Figure 3 b shows the $g^{(2)}(\tau)$-function of same molecule, but under 0-1 excitation. It corresponds to the completely incoherent excitation of the system, with a close-to-zero excitation intensity. Rabi oscillations are not observed, since the incident intensity on the molecule is not sufficient. The fast decay into the $\left|S_{1}\right\rangle$-state can lead to a population inversion. The temporal behavior of the onset of the $g^{(2)}(\tau)$-function reveals to a good approximation the longitudinal lifetime $\left(T_{1}\right.$-time, $\left.=1 / \Gamma 1\right)$ of the molecules excited $\left|S_{1}\right\rangle$-state. This is only valid at the low excitation limit $(k \approx 0)$. The anti-bunching is simply fitted as:

$g^{(2)}(\tau)=1-\mathrm{e}^{|\tau|(-(\Gamma 1+k))}$.

Here, $k$ denotes the pumping rate and is close to zero due to the fast relaxation from the vibrational excited state.

\section{Atomic spectra and Faraday filtering}

Atomic sodium has been studied extensively for more than a century. Due to the availability of narrow-band dye lasers and detectors in the UV-Vis range, the atomic spectroscopy in the past 40 years started with many experiments on

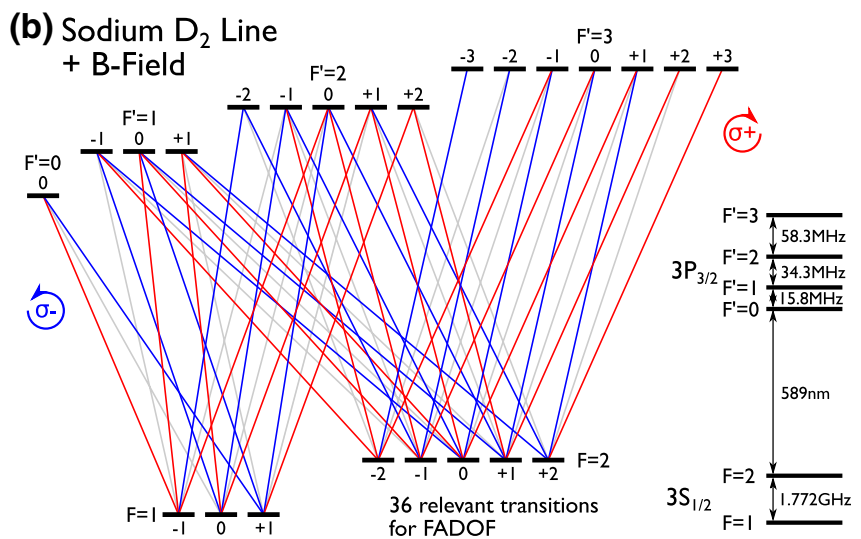

account. Eighteen levels act on left-handed circular polarized light (blue), and corresponding 18 levels act on right-handed polarized light $(r e d)$. All direct vertical transitions do not contribute to the optical rotation and are therefore depicted in gray

atomic vapor cells and atomic beams of sodium, see, for example, [36, 37]. Atomic sodium exhibits a 1.77-GHzwide ground-state splitting, which implies that studies on hot, Doppler-broadened, atomic vapor usually shows one joined transition, in which both transitions are matched. Atomic sodium is well documented [38].

Nowadays, spectroscopy on atomic rubidium is more fashionable, due to the availability of convenient external cavity diode lasers in the near-infrared, the spectral separation of the $D_{1}$ and the $D_{2}$ transitions, and the $6.8-\mathrm{GHz}$ ground-state splitting. The latter implies that both transitions can be individually optically addressed. Rubidium does not require significant heating or excessive magnetic fields. Due to the higher mass, the Doppler broadening is smaller.

Figure $4 \mathrm{a}$ shows the level diagram of atomic sodium. Due to the higher oscillator strength, the $D_{2}$-line transitions are preferred in the following experiments. By Dopplerfree spectroscopy, all levels can be conveniently resolved. The excited-state $D_{1}$-line splitting of $188.8 \mathrm{MHz}$ shows a typical signature in the spectrum. Due to the so-called crossover resonances, 3 sets (distance $\approx 1.77 \mathrm{GHz} / 2$ ) of 3 lines (splitting $\approx 188.8 \mathrm{MHz} / 2$ ) are observed. The $D_{2}$-line exhibits a smaller splitting of the excited state, and the Doppler-free spectroscopy might result in three unresolved groups of transitions. Such a Doppler-free spectrum of the $D_{2}$-line is depicted, for example, in Fig. 7.

For experiments with single photons, which originate from a single molecule, we implemented a sodium Faraday anomalous dispersion optical filter (FADOF) [39-41]. The design is based on an atomic vapor cell between two crossed polarizers. Therefore, no light can simply pass the optical configuration. Only, when a magnetic field is applied, the introduced optical rotation by the Zeeman effect allows the transmission of near-atom-resonant light. 
At the ideal points, such a filter exhibits a transmission close to unity and a GHz-wide transmission spectrum [42]. All other wavelengths, which do not match to an atomic transition, will not pass the filter.

This filter scheme is very interesting to be used in the combination with solid-state single-photon emitters, since all non-resonant emission is efficiently suppressed. Furthermore, the filter is much more stable than temperature-tuned filter cavities. The linewidth depicted in Fig. $2 b$ shows that the spectral selection will not work efficiently in the $0-1$ excitation scheme. An excitation laser might be exciting many different molecules, even at the far-reaching Lorentzian tail. Due to the GHz-large linewidth, the concentration needs to be reduced against usual single-molecule studies. This reduces the background, but it can be further enhanced by the utilization of a FADOF. The filter allows to reach very high peak transmission and a small background contribution. The crucial quantity for this is the so-called equivalent noise band width (ENBW). This is calculated by the integrated transmission, $T$, for all relevant wavelengths, divided by the peak transmission at the point of maximal transmission $\left(\nu_{\mathrm{S}}\right)$, as ENBW $=\frac{\int T(\nu) \mathrm{d} v}{T\left(\nu_{\mathrm{S}}\right)}$. It describes the peak to background ratio and amounts to below $5 \mathrm{GHz}$ in the case for the filter on the sodium $D_{2}$-line [42-44].

The spectrum of such a FADOF filter can be relatively straightforwardly calculated, by evaluating the electric susceptibilities of the atomic vapor. This requires to add the weighted contribution of all transitions. For the $D_{1}$-line, these are 24 transitions, whereas for the $D_{2}$-line, 36 transitions have to be taken into account. For the latter case, the relevant transitions are depicted in Fig. 4b. Eighteen transitions act on the $\sigma+$ components of the incident light and 18 transitions on the $\sigma-$ component. All direct vertical transitions do not contribute to the optical rotation and are therefore depicted in gray.

The calculation requires a diagonalization of the full Hamiltonian, which is composed of three parts, including the hyperfinesplitting (HFS) and the Zeeman term. This is due to the fact that the filter is not operated solely in the low- or high-field approximation regime. It is calculated as $H=H_{0}+H_{\mathrm{HFS}}+H_{\text {Zeeman }}$. This results in the complex susceptibilities $\chi_{ \pm}=\chi_{ \pm}^{\prime}+i \chi_{ \pm}^{\prime \prime}$, in which the + and - signs denote the susceptibility per circular polarization direction. Together with the length of the cell, $L$, and the temperature, a Voigt line profile is obtained. The combination of optical rotation by $\pi / 2$ and simultaneous weak Doppler absorption leads to a preferred setting for the cell temperature and the magnetic field. The transmission, $T$, is calculated as

$$
\begin{aligned}
T= & 1 / 4\left[\exp \left(-\frac{\omega}{c} \chi_{+}^{\prime \prime} L\right)+\exp \left(-\frac{\omega}{c} \chi_{-}^{\prime \prime} L\right)\right. \\
& \left.-2 \exp \left(-\frac{\omega}{c} \frac{\chi_{+}^{\prime \prime}+\chi_{-}^{\prime \prime}}{2} L\right) \cdot \cos \left(\frac{\omega}{c} \frac{\chi_{+}^{\prime}-\chi_{-}^{\prime}}{2} L\right)\right] .
\end{aligned}
$$

We performed the full calculation. The source and the data are available as supporting material online [45]. Another convenient way of calculating the relevant filter functions is the use of the program "ElecSus" by the Durham atomic physics group [46].

For an experimental confirmation, an atomic vapor cell with an optical path length of $100 \mathrm{~mm}$ is placed in a solenoid configuration, capable of generating up to $400 \mathrm{mT}$ magnetic field. The exact experimental configuration is described elsewhere [42].

Figure 5 shows a series of $D_{2}$-line spectra of atomic sodium at a given temperature $\left(152{ }^{\circ} \mathrm{C}\right)$. For each panel on the horizontal axis, the frequency detuning from the center of mass is depicted. The vertical axes shows the spectrum against an increasing magnetic field. The three 2D spectra which are abreast show the measurement of the following three entities: left The FADOF spectrum, in which the input and the output polarizers are orthogonal. middle The crossed polarization, i.e., when the input and output polarizer are aligned with each other. We call this the cross-FADOF (X-FADOF) spectrum. right When both the FADOF and the X-FADOF spectra are added, it corresponds to the simple Doppler absorption spectrum as it would be measured with unpolarized light. The theoretical spectra are on the top panel (a), and experimental data are displayed in the bottom panel (b).

First, the FADOF spectrum is depicted. As it can be clearly seen, right and left of the center, the transmission goes to zero, since both polarizers in front and behind the cell are crossed out. Without a magnetic field, no transmission occurs. Only with an increased magnetic field, some transmission arises. The spectral components are approx. linearly shifted due to the Zeeman effect. From approx. $150 \mathrm{mT}$, the two groups, both acting on one part of the circular decomposed linear polarization, are separated, such that the simple absorption of the cell is diminished. Only then, the transmission arises above $50 \%$ and reaches to values above $90 \%$ [42]. This is considered as a preferred point for further measurements in combination with single photons from a DBATT molecule and atomic Faraday filters.

In the middle row, the X-FADOF spectrum displays the other output port of the analyzing polarizing beam splitter. Since both polarizers are aligned with each other, far from the atomic resonance the transmission amounts to $100 \%$. Without a magnetic field, the spectrum corresponds to the Doppler spectrum. At the optimal working conditions of the Faraday filter (150-200 mT), the rotation in the vapor amounts to $90^{\circ}$, such that the X-FADOF spectrum displays a minimum on resonance. Acquiring the data for the $\mathrm{X}$-FADOF gives an advantage over recording the FADOF spectrum alone, since both components can be individually determined and allow a good comparison with the theory. 

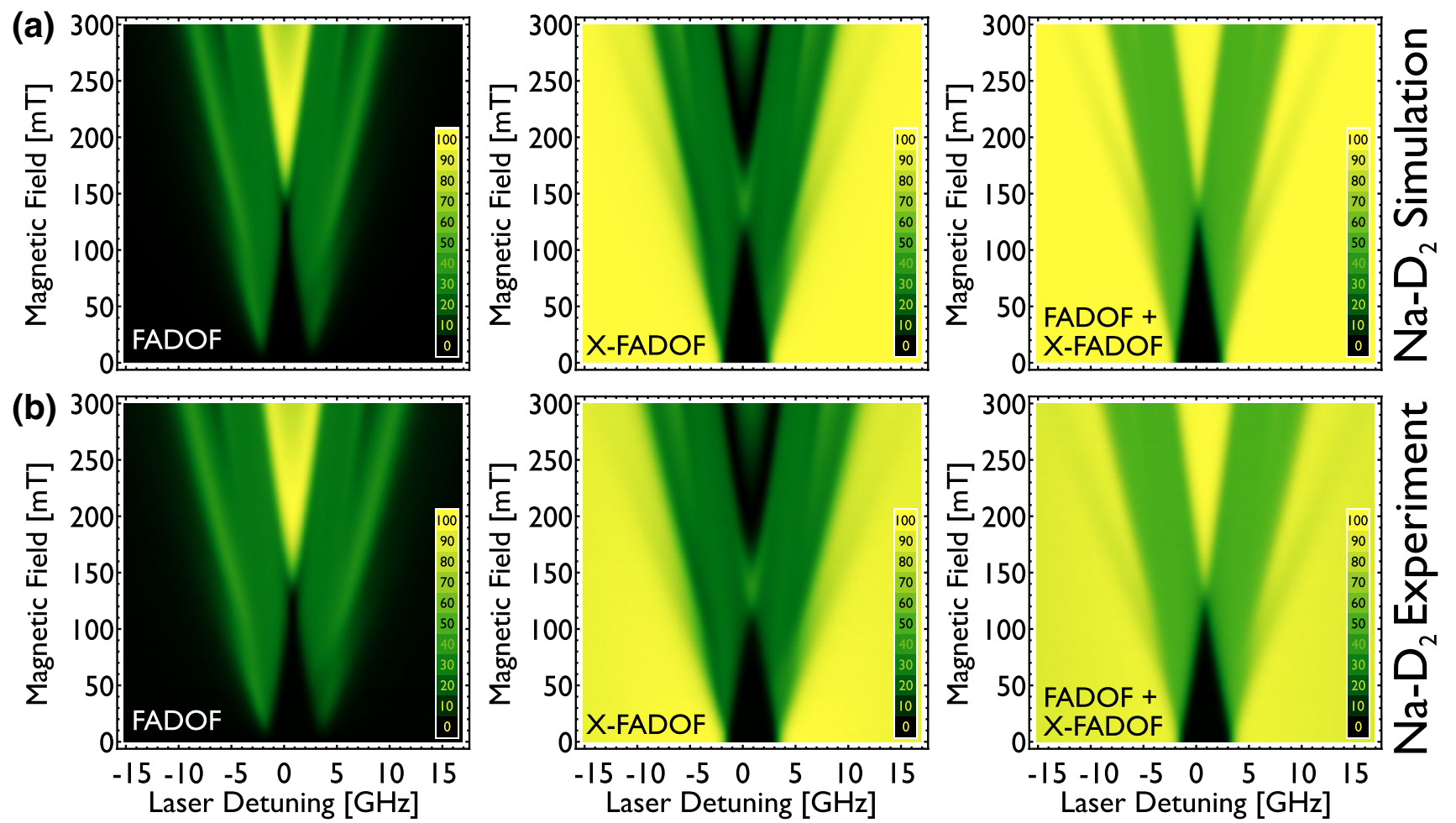

Fig. 5 Simulated and measured spectra, acquired with a dye laser at a cell temperature of $152{ }^{\circ} \mathrm{C}$. Cell length $100 \mathrm{~mm}$. a Simulation of both measured output ports (FADOF and X-FADOF), and an addition of both ports, which simply represents the Doppler spectrum under the influence of a magnetic field. b Same as above, but experimental

The sum of the FADOF and the X-FADOF simply results in the Doppler absorption spectrum (right panel). At zero magnetic field, this reaches down to very small values, since the optical density is a few orders of magnitude. When a magnetic field is applied, both the $\sigma+$ and the $\sigma-$ components are differently affected, and the overall transmission is diminished to approx. $50 \%$ as in the case of the FADOF spectrum. The Doppler spectrum at zero magnetic field allows to again determine the exact temperature of the sodium vapor with very simplified assumptions and no full calculation of the susceptibilities.

We find an excellent match between the simulated spectra (Fig. 5a) and the experimental data (Fig. 5b). The frequency scale of the experimental data represents the full scan range of the utilized laser. With optimized settings, it is possible to extend the mode-hop-free scan range of the laser up to $34 \mathrm{GHz}$. The frequency axes was calibrated by Doppler-free sodium spectroscopy. An increasing background per scan was accounted for, since the laser was changing the emitted output power approx. linearly during the scan. The transmission of the cell, which was measured to amount to approx. $85 \%$, was neglected, i.e., the scale was adapted to compensate for the loss of the cell windows. data. The overall transmission of the cell was adapted, due to finite absorption of our sodium cell. Also, the spectrum is slightly broadened due to a higher cell temperature and a slightly larger Doppler linewidth of the atoms. The maximum current was approx. $200 \mathrm{~A}$

\section{Combination of single molecule and atomic spectroscopy}

The narrow-band single-photon emission of a single molecule under 0-1 excitation is now interlinked with the presented atomic Faraday filter. A scheme of the experimental configuration is shown in Fig. 6. The excitation light is generated by a dye-ring laser (Coherent 899-29) with the dye Rhodamine $6 \mathrm{G}$. The beam is reflected via a quartz wedge ( $\mathrm{R}: \mathrm{T}=10: 90)$ into a He bathcryostat (Janis). For efficient excitation and collection to and from the molecule, a hemispherical solid immersion lens (SIL) made of cubic Zirconia $\left(\mathrm{ZrO}_{2} \varnothing=3 \mathrm{~mm}, n=2.18\right)$ is used in combination with an aspheric lens (Geltech C330). This configuration is commonly used in single-emitter spectroscopy [12, $47,48]$. Beam scanning is possible, but results in an accessible area of approx. $50 \times 50 \mu \mathrm{m}^{2}$, since otherwise the $1 / 4 \lambda$-criterion is not fulfilled [49] and aberration leads to a low collection efficiency. For the $0-1$ excitation experiments, where the narrow-band photons on or close to the sodium line are detected, the light is collected transmitted through the quartz wedge and filtered by a $589-\mathrm{nm}$ approx. 1-nm-wide band-pass filter (Omega Optical). This ensures 


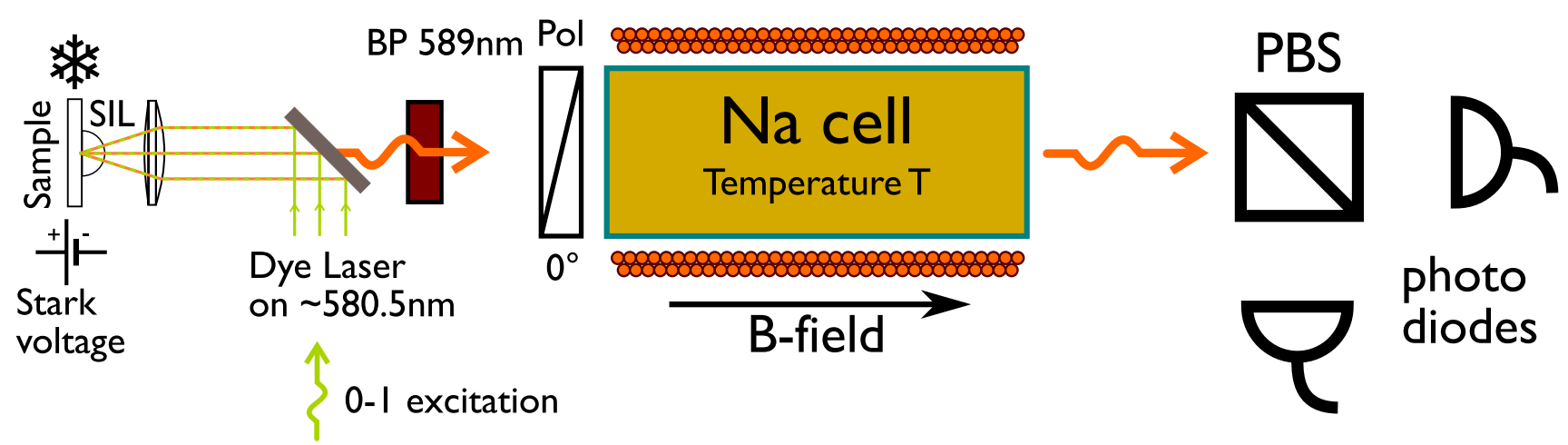

Fig. 6 Scheme of the experimental configuration. A dye-ring laser at approximately $580 \mathrm{~nm}$ is focused on the sample of single molecules in an organic matrix. The sample resides in a He bathcryostat at $T=1.4 \mathrm{~K}$. For optimal light extraction, the optics consists of a solid immersion lens and a single asphere. The light is then collected

that only the resonant photons and not the Stokes-shifted emission is passed through the cell. To block the scattered light off the sample, another 585-nm long-pass filter (Omega Optical XF3090, not shown) is added, which helps to reduce the background due to the high-power 0-1 excitation scheme even more. For experiments under 0-0 excitation, the 594LP filter is used. The atomic cell can stay in place, since the sodium-resonant photons are not detected under 0-0 excitation.

After this coarse spectral filtering of the photons, the photons are passed through an achromatic half-wave plate (omitted in Fig. 6) and a linear polarizer. Then, the vapor cell in a solenoid and a second polarizer form the Faraday filter. The photons are then focused onto an avalanche photodiode (APD, SPCM-AQR, Excellitas). No further spatial selection, such as a pinhole, is introduced in the beam path. The X-FADOF path was not used in this experiment with the molecule, due to space constraints in the optical setup behind the atomic vapor cell.

For the experiment, single photons close to the sodium wavelength are required. The inhomogeneous broadening of the single-molecule transitions usually allows to find a spectral match between an individual single molecule and atomic sodium transition in a highly concentrated sample. This match is always initially verified under $0-0$ excitation. A high concentration implies that many molecules might have a spectral overlap or exhibit long spectral tails, which scale as a Lorentzian as $1 / \delta^{2}$, with $\delta$ being the detuning from the line center. Therefore, the single-molecule singlephoton emission might be spoiled by other (weakly) emitting molecules. This increases the background and reduces the purity of the further used single photons. The electronic transition of a single molecule can be conveniently detuned by the DC Stark effect [50-53], and therefore, a lower on the same way back, and the polarization is fixed by a Glan-Laser polarizer. The light passes through an atomic vapor cell, and both polarization components are detected. This is usually performed with single-photon detectors, but for characterizing the filter by a laser, these were replaced by normal photodiodes

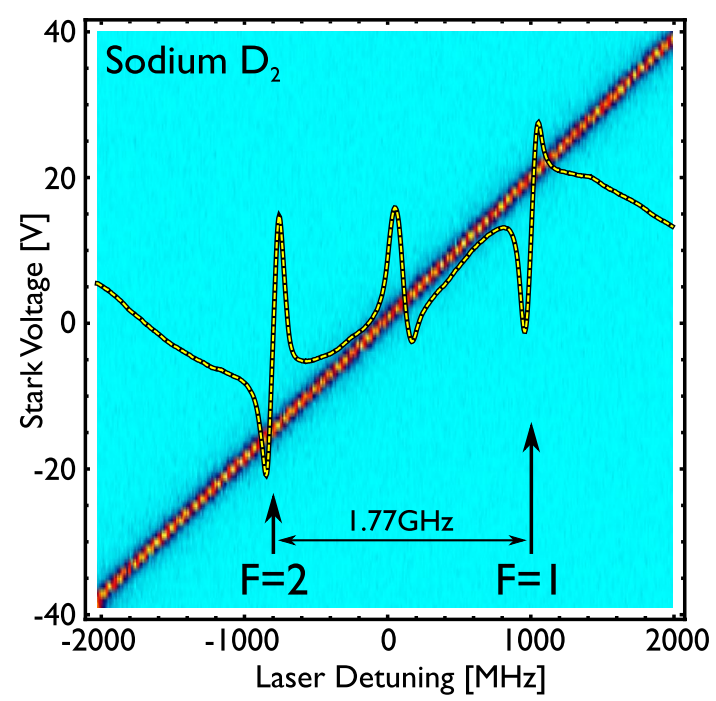

Fig. 7 Stark scan of a single molecule under 0-0 excitation. By interdigitating electrodes, the spectral position of the molecules zero-phonon line is changed. A linear DC Stark effect is observed. In parallel, the Doppler-free spectrum of sodium is recorded. This shows that the molecules spectral transition can be deliberately changed across the entire sodium spectrum. Small intensity fluctuations are caused by a fluctuating laser intensity in conjunction with a short $(2 \mathrm{~ms})$ integration time

concentration of molecules can be used in the sample. When a molecule is close (i.e., within a few $\mathrm{GHz}$ ) to the desired transition, the electronic transition can be spectraltuned to the desired point.

To spectral-detune the molecules, we used interdigitating gold electrodes with $25 \mu \mathrm{m}$ width and $25 \mu \mathrm{m}$ spacing. The electrodes are electrically isolated with a thin, sputtered $\mathrm{SiO}_{2}$ film, since the solid immersion lens is held in place by a thin brass metal sheet, which would otherwise 
shorten the electrical contacts. The full sample was subsequently assembled of: a glass plate with the electrodes, the $n$-tetradecane solution, doped by DBATT molecules, the solid immersion lens, and a brass sheet metal which was held in place with two small magnets (IBS Magnet Berlin). The voltage is provided by a bipolar high-voltage power supply module (ultravolt TN-HVA-1), which can be conveniently controlled by the analog output voltage of a data acquisition card in the computer. The output voltage reaches between -1000 and $1000 \mathrm{~V}$. For the utilized electrodes, voltages below $-300 \mathrm{~V}$ and above $300 \mathrm{~V}$ should not be used, since the electrodes show the tendency of an electric breakdown.

Figure 7 shows a Stark scan of a single molecule across the Doppler-free spectrum of atomic sodium. Every horizontal line represents a 0-0 sweep of the excitation laser around the sodium wavelength. When the molecule is on resonance, the amount of emitted photons is increased (red, yellow line in density plot). Vertically, the voltage across the electrodes is changed. The observed, linear Stark effect shows a change of $50 \mathrm{MHz}$ per $1 \mathrm{~V}$ across the electrodes. Nominally, this $1 \mathrm{~V}$ corresponds to a field of $40 \mathrm{kV} / \mathrm{m}$. This might only be an approximation, since the affected molecule does not necessarily be in the plane with the electrodes, but might be some micrometers above the electrode level. In this case, we observe a linear Stark effect, as it was reported in the literature before [53]. In other experiments with the same settings, we also observed quadratic Stark parabola in the same host matrix system with DBATT molecules. We assume that this behavior can be explained by small differences in the matrix structure. With the selected molecule, all spectral positions across the sodium resonance can be reached. The molecules linewidth is not affected. With every spectral scan, a Doppler-free spectrum of atomic sodium is recorded. Since this acts as a very stable frequency reference, which supersedes the accuracy of the lasers internal reference cavity, the acquired atomic spectra could act as a long-term stable reference. The slight intensity change in the laser when scanning does not strongly affect the recorded 0-0 spectra, since the scan is only over $4 \mathrm{GHz}$, unlike the corrected laser spectra in Fig. 5.

The same molecule can then be used as a narrow-band single-photon source. For this, the excitation scheme is changed to 0-1 excitation, where many narrow-band photons are emitted. The experimental setup selects only the near-sodium-resonant photons by the $585-\mathrm{nm}$ long-pass and the 589-nm band-pass filter. The excitation laser is tuned to $17226.1 \mathrm{~cm}^{-1}$ as in Fig. $2 \mathrm{~b}$. The emitted photons cannot pass the FADOF without any magnetic field, since the atomic vapor absorbs all resonant photons. Therefore, the current on the solenoid is set to approx. $125 \mathrm{~A}$, which corresponds to $200 \mathrm{mT}$. When the emission is observed, the focal position is optimized for the small spectral shift. The lateral focus position is thereafter also optimized by simple beam scanning.

When now the electric field across the electrodes is modified, the molecule undergoes the same DC Stark effect, which was observed under 0-0 excitation, as shown in Fig. 7. Therefore, the narrow-band emission of the molecules is detuned. An uncorrected recording of the FADOF spectrum, acquired only with single photons originating from a single molecule, is shown in Fig. 8. The spectrum resembles the predicted calculation and shows a peak flux of $9.9 \times 10^{4}$ counts per second. Due to the narrow spectral linewidth of the molecule, the further broadening of the spectrum is negligible. The scan range of the molecular emission spans more than $20 \mathrm{GHz}$ with the utilized electrodes. This range can be massively increased with smaller and better isolated electrodes. Unfortunately, with very high fields, the spectral stability of the molecule might be affected [54].

With the spectrum, we find an increased background contribution when the voltage is increased. This is likely another molecule, which is initially weakly excited, but contributes at $+250 \mathrm{~V}$ to approx. $7 \%$ of the counts against the expected spectrum. This background contribution is assumed to be a long Lorentzian tail, but is depicted in Fig. 8 as a linear increasing background. Such a contribution can be suppressed, when a lower concentration of dopant molecules is present in the host matrix. This implies of course a reduced probability of finding a suitable molecule. This is always the dilemma of the $0-1$ excitation experiments.

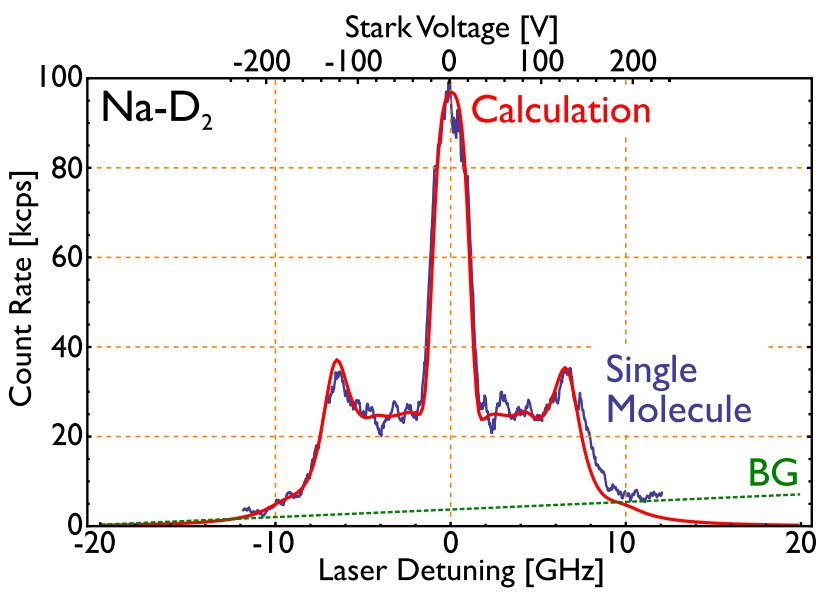

Fig. 8 A scan of the Stark voltage with a single molecule in the focus under 0-1 excitation. Depicted is the count rate of the single molecule, which is observed through the Faraday filter on a singlephoton detector. The Stark voltage was changed from -250 to $250 \mathrm{~V}$. Due to the spectral linewidth of the molecules under this excitation scheme, the background depends on the Stark voltage on the sample. The green line represents the increasing background 

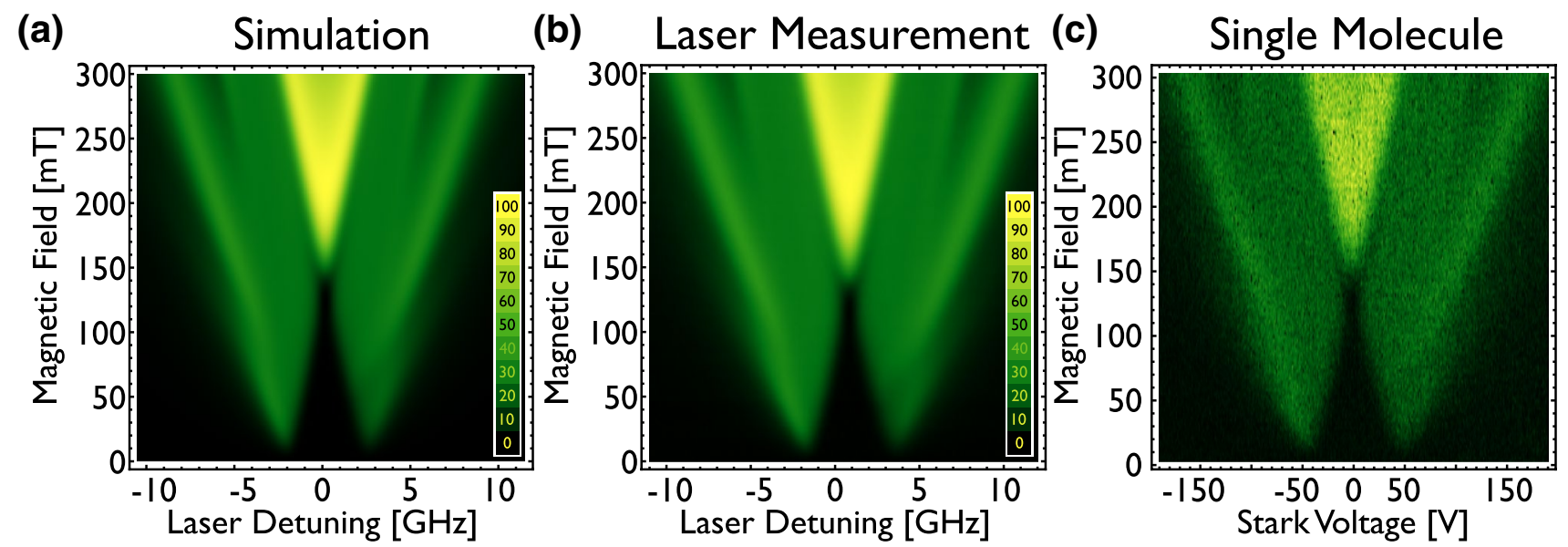

Fig. 9 Spectrum of the sodium $D_{2}$-line Faraday anomalous dispersion optical filter with different magnetic fields at a cell temperature of $153{ }^{\circ} \mathrm{C}$. a Simulation for $153{ }^{\circ} \mathrm{C}$, b measurement with the dye laser, the transmission axes are scaled to compensate for the finite transmission of the atomic cell. c Measurement with a single-mol-

Since the molecules are very stable and do not exhibit any blinking or bleaching, it is possible to acquire a full scan of the Faraday filter with the above-selected molecule. To reduce background, the 0-1 excitation intensity was reduced. Subsequently, the peak power in Fig. 9 amounts to $71 \mathrm{kcps}$. We recorded the Stark detuning with 1000 different voltages from -250 to $250 \mathrm{~V}(0.5 \mathrm{~V}$ per step $)$ and 120 different magnetic fields from 0 to $350 \mathrm{mT}$. For clarity, and to match our earlier simulation and experimental data, which was acquired with a laser, we only show a part of the data. The integration time per pixel was $5 \mathrm{~ms}$. The nominal recording time was subsequently $600 \mathrm{~s}$, but the entire scan time was approx. $1000 \mathrm{~s}$ due to overhead by the measurement programs. Many of these scans were recorded over the course of several hours.

\section{Conclusion and outlook}

This paper introduced the different excitation schemes of a single molecule. The light can be conveniently filtered by an atomic Faraday filter (FADOF).

Of course, the presented Faraday filter spectra with a single-molecule light source do not differ by any means from the spectra which are acquired in a more convenient laser configuration. More importantly, the presented spectra show the universality of the experimental configuration, the spectral long-term stability of the molecules, their enormous brightness, and their simultaneous narrowband nature. No further studies on the propagation delay have been performed in this configuration. This might be ecule light source. Unlike in Fig. 8, the maximum count rate corresponds to $7.1 \times 10^{4}$ counts per second. The recording with the single molecule is recorded to higher magnetic fields than the spectra in a and $\mathbf{b}$. Please note that the plot represents raw data, which was scaled according to the changing overall emission due to the Stark scan

an interesting next step, when the different group velocity delay affects the single photons which are passing the vapor cell.

The presented single-photon source has a variety of applications, since it allows to interlink different types of spectroscopy. For example, single photons might be efficiently generated by a single molecule and be filtered by an atomic Faraday filter and then interact with an atomic vapor, e.g., utilizing higher Rydberg states. Such photons might then interact again with a single molecule, since the efficient interaction of resonant photons with a molecule has been proven earlier [47, 55, 56]. Experiments with the free-space daylight transmission of single photons might gain by the suppression of the sun's radiation by the Fraunhofer lines. Such experiments were conducted earlier by the NIST QKD group [57]. The sodium Fraunhofer lines are comparable "dim" as the H-alpha line. The use of a Faraday filter, which again reduces the background, could again reduce the sun's background contribution [58].

Unlike atomic sources, the presented molecular source can be quickly spectral-detuned by several GHz. The speed is in our case limited by the utilized high-voltage power supply and the throughput capacitance of the wiring into the cryostat [59]. The likely unsurpassed efficiency of light extraction of a single molecule [11] might allow for the generation of higherorder Fock states, when several molecules are spectral-tuned on resonance inside a focal volume. This requires well-isolated micro-electrodes and will be challenging.

We presently plan further experiments which interlink the spectroscopy on a cold atomic vapor, e.g., in a magnetooptical trap, with the photons originating from a molecule. 
Acknowledgments Open access funding provided by Max Planck Society. We acknowledge the work performed by P. Siyushev and G. Stein who started the experiments with the combination of single DBATT molecules and atomic sodium. We further acknowledge the funding from the MPG via a Max Planck fellowship (J.W.), the SFB project CO.CO.MAT/TR21, the Bundesministerium für Bildung und Forschung (BMBF), the project Q.COM, and SQUTEC.

Open Access This article is distributed under the terms of the Creative Commons Attribution 4.0 International License (http://creativecommons.org/licenses/by/4.0/), which permits unrestricted use, distribution, and reproduction in any medium, provided you give appropriate credit to the original author(s) and the source, provide a link to the Creative Commons license, and indicate if changes were made.

\section{References}

1. I. Gerhardt, G. Wrigge, G. Zumofen, J. Hwang, A. Renn, V. Sandoghdar, Coherent state preparation and observation of rabi oscillations in a single molecule. Phys. Rev. A 79(1), 011402 (2009)

2. H.J. Kimble, M. Dagenais, L. Mandel, Photon antibunching in resonance fluorescence. Phys. Rev. Lett. 39, 691-695 (1977)

3. W. Neuhauser, M. Hohenstatt, P.E. Toschek, H. Dehmelt, Localized visible $\mathrm{Ba}^{+}$mono-ion oscillator. Phys. Rev. A 22, 11371140 (1980)

4. F. Diedrich, H. Walther, Nonclassical radiation of a single stored ion. Phys. Rev. Lett. 58, 203-206 (1987)

5. J.T. Höffges, H.W. Baldauf, T. Eichler, S.R. Helmfrid, H. Walther, Heterodyne measurement of the fluorescent radiation of a single trapped ion. Opt. Commun. 133(16), 170-174 (1997)

6. B. Lounis, M. Orrit, Single-photon sources. Rep. Prog. Phys. 68(5), 1129 (2005)

7. L. Novotny, B. Hecht, Principles of Nano-Optics (Cambridge University Press, Cambridge, 2012)

8. W. Alt, An objective lens for efficient fluorescence detection of single atoms. Optik 113, 142 (2002)

9. L.M. Bennie, P.T. Starkey, M. Jasperse, C.J. Billington, R.P. Anderson, L.D. Turner, A versatile high resolution objective for imaging quantum gases. Opt. Express 21(7), 9011-9016 (2013)

10. W. Lukosz, R.E. Kunz, Light emission by magnetic and electric dipoles close to a plane dielectric interface. II. Radiation patterns of perpendicular oriented dipoles. J. Opt. Soc. Am. 67(12), 1615-1619 (1977)

11. K. Lee, X. Chen, H. Eghlidi, P. Kukura, R. Lettow, A. Renn, V. Sandoghdar, S. Götzinger, A planar dielectric antenna for directional single-photon emission and near-unity collection efficiency. Nat. Photonics 5(3), 166-169 (2011)

12. W.L. Barnes, G. Björk, J.M. Gérard, P. Jonsson, J.A.E. Wasey, P.T. Worthing, V. Zwiller, Solid-state single photon sources: light collection strategies. Eur. Phys. J. D 18, 197-210 (2002)

13. S. Ali Momenzadeh, R.J. Stöhr, F.F. de Oliveira, A. Brunner, A. Denisenko, S. Yang, F. Reinhard, J. Wrachtrup, Nanoengineered diamond waveguide as a robust bright platform for nanomagnetometry using shallow nitrogen vacancy centers. Nano Lett. 15(1), 165-169 (2015)

14. I. Gerhardt, G. Wrigge, J. Hwang, G. Zumofen, V. Sandoghdar, Coherent nonlinear single molecule microscopy. Phys. Rev. A 82(6), 063823 (2010)

15. P. Neumann, I. Jakobi, F. Dolde, C. Burk, R. Reuter, G. Waldherr, J. Honert, T. Wolf, A. Brunner, J.H. Shim, D. Suter, H. Sumiya, J. Isoya, J. Wrachtrup, High-precision nanoscale temperature sensing using single defects in diamond. Nano Lett. 13(6), 2738-2742 (2013)
16. A. Kuhn, M. Hennrich, G. Rempe, Deterministic single-photon source for distributed quantum networking. Phys. Rev. Lett. 89, 067901 (2002)

17. S. Ritter, C. Nölleke, C. Hahn, A. Reiserer, A. Neuzner, M. Uphoff, M. Mücke, E. Figueroa, J. Bochmann, G. Rempe, An elementary quantum network of single atoms in optical cavities. Nature 484, 195 (2012)

18. N. Akopian, L. Wang, A. Rastelli, O.G. Schmidt, V. Zwiller, Hybrid semiconductor-atomic interface: slowing down single photons from a quantum dot. Nat. Photonics 5, 230-233 (2011)

19. P. Siyushev, G. Stein, J. Wrachtrup, I. Gerhardt, Molecular photons interfaced with alkali atoms. Nature 509(7498), 66-70 (2014)

20. S.M. Ulrich, S. Weiler, M. Oster, M. Jetter, A. Urvoy, R. Löw, P. Michler, Spectroscopy of the $D_{1}$ transition of cesium by dressedstate resonance fluorescence from a single (In, Ga)As/GaAs quantum dot. Phys. Rev. B 90, 125310 (2014)

21. W.E. Moerner, L. Kador, Optical detection and spectroscopy of single molecules in a solid. Phys. Rev. Lett. 62, 2535-2538 (1989)

22. M. Orrit, J. Bernard, Single pentacene molecules detected by fluorescence excitation in a $p$-terphenyl crystal. Phys. Rev. Lett. 65, 2716-2719 (1990)

23. S. Kummer, T. Basch, C. Bräuchle, Terrylene in $p$-terphenyl: a novel single crystalline system for single molecule spectroscopy at low temperatures. Chem. Phys. Lett. 229(3), 309-316 (1994)

24. S. Mais, J. Tittel, T. Basché, C. Bräuchle, W. Ghde, H. Fuchs, G. Müller, K. Müllen, Terrylenediimide: a novel fluorophore for single-molecule spectroscopy and microscopy from $1.4 \mathrm{~K}$ to room temperature. J. Phys. Chem. A 101(45), 8435-8440 (1997)

25. A.L. Aurlien Nicolet, P. Bordat, C. Hofmann, M.A. Kol'chenko, B. Kozankiewicz, R. Brown, M. Orrit, Single dibenzoterrylene molecules in an anthracene crystal: main insertion sites. ChemPhysChem 8(13), 1929-1936 (2007)

26. M. Pirotta, A. Renn, M.H.V. Werts, U.P. Wild, Single molecule spectroscopy. Perylene in the Shpol'skii matrix $n$-nonane. Chem. Phys. Lett. 250(56), 576-582 (1996)

27. P. Navarro Pérez, Stable single molecules for quantum optics and all-optical switches. Ph.D. Thesis, Leiden Institute of Physics (LION), Faculty of Science, Leiden University (2014)

28. A.-M. Boiron, B. Lounis, M. Orrit, Single molecules of dibenzanthanthrene in $n$-hexadecane. J. Chem. Phys. 105(10), 39693974 (1996)

29. I. Gerhardt, Scattering and absorption of light by a single molecule under a subwavelength aperture. Ph.D. Thesis, ETH Zurich (2006). doi:10.3929/ethz-a-005279519

30. T. Plakhotnik, E.A. Donley, U.P. Wild, Single-molecule spectroscopy. Annu. Rev. Phys. Chem. 48(1), 181-212 (1997)

31. T.Y. Latychevskaia, Single molecules in electric fields. Ph.D. Thesis, ETH Zurich (2002)

32. J. Sepiol, A. Starukhin, R. Koos, T. Latychevskaia, J. Jasny, A. Renn, U.P. Wild, Detection and spectroscopy of single molecules in rare gas matrices: dibenzanthanthrene in krypton and xenon. Chem. Phys. Lett. 311(12), 29-35 (1999)

33. R.J. Pfab, J. Zimmermann, C. Hettich, I. Gerhardt, A. Renn, V. Sandoghdar, Aligned terrylene molecules in a spin-coated ultrathin crystalline film of $p$-terphenyl. Chem. Phys. Lett. 387, 490-495 (2004)

34. G.G.C. Cohen-Tannoudji, J. Dupont-Roc, Atom-Photon Interactions: Basic Process and Appilcations (WILEY-VCH Verlag $\mathrm{GmbH} \& \mathrm{Co}, \mathrm{KGaA}, 2004)$

35. T. Basché, W.E. Moerner, M. Orrit, H. Talon, Photon antibunching in the fluorescence of a single dye molecule trapped in a solid. Phys. Rev. Lett. 69, 1516-1519 (1992)

36. J.E. Thomas, P.R. Hemmer, S. Ezekiel, C.C. Leiby, R.H. Picard, C.R. Willis, Observation of ramsey fringes using a stimulated, 
resonance raman transition in a sodium atomic beam. Phys. Rev. Lett. 48, 867-870 (1982)

37. B.W. Peuse, M.G. Prentiss, S. Ezekiel, Observation of resonant light diffraction by an atomic beam. Phys. Rev. Lett. 49, 269272 (1982)

38. D.A. Steck, Sodium D line data. Technical report, University of Oregon (2010)

39. Y. Öhman, On some new auxiliary instruments in astrophysical research. Stockholms observatoriums annaler (1956)

40. D.J. Dick, T.M. Shay, Ultrahigh-noise rejection optical filter. Opt. Lett. 16(11), 867-869 (1991)

41. S.D. Harrell, C.-Y. She, T. Yuan, D.A. Krueger, H. Chen, S.S. Chen, Z.L. Hu, Sodium and potassium vapor faraday filters revisited: theory and applications. J. Opt. Soc. Am. B 26(4), 659-670 (2009)

42. W. Kiefer, R. Löw, J. Wrachtrup, I. Gerhardt, Na-faraday rotation filtering: the optimal point. Sci. Rep. 4, 6552 (2014). doi:10.1038/srep06552

43. M.A. Zentile, D.J. Whiting, J. Keaveney, C.S. Adams, I.G. Hughes, Atomic faraday filter with equivalent noise bandwidth less than 1 GHz. Opt. Lett. 40(9), 2000-2003 (2015)

44. M. Widmann, S. Portalupi, S.-Y. Lee, P. Michler, J. Wrachtrup, I. Gerhardt, Faraday filtering on the Cs- $\mathrm{D}_{1}$-line for quantum hybrid systems (2015). arXiv:1505.01719

45. I. Gerhardt, Sodium susceptibilities with Mathematica. Webpage (2015)

46. M.A. Zentile, J. Keaveney, L. Weller, D.J. Whiting, C.S. Adams, I.G. Hughes, ElecSus: a program to calculate the electric susceptibility of an atomic ensemble. Comput. Phys. Commun. 189, 162-174 (2015)

47. G. Wrigge, I. Gerhardt, J. Hwang, G. Zumofen, V. Sandoghdar, Efficient coupling of photons to a single molecule and the observation of its resonance fluorescence. Nat. Phys. 4(1), 60-66 (2008)

48. M. Jamali, I. Gerhardt, M. Rezai, K. Frenner, H. Fedder, J. Wrachtrup, Microscopic diamond solid-immersion-lenses fabricated around single defect centers by focused ion beam milling. Rev. Sci. Instrum. 85(12), 123703 (2014)
49. M. Baba, T. Sasaki, M. Yoshita, H. Akiyama, Aberrations and allowances for errors in a hemisphere solid immersion lens for submicron-resolution photoluminescence microscopy. J. Appl. Phys. 85(9), 6923-6925 (1999)

50. U.P. Wild, F. Güttler, M. Pirotta, A. Renn, Single molecule spectroscopy: stark effect of pentacene in $p$-terphenyl. Chem. Phys. Lett. 193(6), 451-455 (1992)

51. M. Orrit, J. Bernard, A. Zumbusch, R.I. Personov, Stark effect on single molecules in a polymer matrix. Chem. Phys. Lett. 196(6), 595-600 (1992)

52. Y.T. Latychevskaia, A. Renn, U.P. Wild, Higher-order stark effect on single-molecules. Chem. Phys. 282(1), 109-119 (2002)

53. R. Lettow, V. Ahtee, R. Pfab, A. Renn, E. Ikonen, S. Götzinger, V. Sandoghdar, Realization of two Fourier-limited solid-state single-photon sources. Opt. Express 15(24), 15842-15847 (2007)

54. I. Gerhardt, G. Wrigge, V. Sandoghdar, Control and imaging of single-molecule spectral dynamics using a nano-electrode. Mol. Phys. 107, 1975-1979 (2009)

55. I. Gerhardt, G. Wrigge, P. Bushev, G. Zumofen, M. Agio, R. Pfab, V. Sandoghdar, Strong extinction of a laser beam by a single molecule. Phys. Rev. Lett. 98, 033601 (2007)

56. Y.L.A. Rezus, S.G. Walt, R. Lettow, A. Renn, G. Zumofen, S. Götzinger, V. Sandoghdar, Single-photon spectroscopy of a single molecule. Phys. Rev. Lett. 108, 093601 (2012)

57. D.J. Rogers, J.C. Bienfang, A. Mink, B.J. Hershman, A. Nakassis, X. Tang, L. Ma, D.H. Su, C.J. Williams, C.W. Clark, Freespace quantum cryptography in the $\mathrm{H}$-alpha Fraunhofer window. Proc. SPIE 6304, 630417 (2006)

58. X. Shan, X. Sun, J. Luo, Z. Tan, M. Zhan, Free-space quantum key distribution with Rb vapor filters. Appl. Phys. Lett. 89(19), 191121 (2006). doi:10.1063/1.2387867

59. C. Brunel, P. Tamarat, B. Lounis, J. Plantard, M. Orrit, Driving the Bloch vector of a single molecule: towards a triggered single photon source. Comptes Rendus de l'Acadmie des Sciences - Series IIB - Mechanics-Physics-Astronomy 326(12), 911-918 (1998) 